\title{
Brecha digital e inclusión digital: fenómenos socio - tecnológicos
}

\author{
LuZ ARABANy Ramírez CASTAÑEDA ${ }^{1}$ \\ JHEIMER JULiáN SEPÚlVEdA LóPEZ ${ }^{1}$
}

\section{Resumen}

Existe una diferencia entre comunidades respecto al acceso y uso de herramientas tecnológicas para satisfacer necesidades y mejorar condiciones de vida; fenómeno definido como brecha digital. Para reducir dicha diferencia se emprenden propuestas y procesos en el marco del fenómeno denominado inclusión digital.

Se ha identificado que estos dos fenómenos integran aspectos sociales y tecnológicos que deben ser considerados al momento de implementar los programas y proyectos.

Adicionalmente, se considera que la Administración de Sistemas Informáticos es un área disciplinar que integra conocimientos necesarios para estudiar dichos fenómenos. La administración es una disciplina de las ciencias sociales y los sistemas informáticos son productos que se desarrollan en el marco de las ciencias exactas.

En el presente documento se plantea la relación entre la Administración de Sistemas Informáticos y el estudio (análisis, mejora, diseño y propuesta) de los fenómenos de la brecha e inclusión digital.

Palabras clave: inclusión digital, brecha digital, sistemas informáticos.

\section{Digital divide and digital inclusion: socio - technological phenomena}

\section{Abstract}

There seems to be a difference among diverse communities, regarding the access and the use of technological tools, to satisfy necessities and to improve the quality of life of people, which is a phenomenon defined as: digital gap. To reduce the gap, there have been carried out several proposals and processes, by means of the digital inclusion phenomenon.

1 Universidad Nacional de Colombia, Sede Manizales, Colombia.

Autor de correspondencia: Sepulveda López, J.J. (Jheimer Julián): Maltería, Bloque 3 \# 31-07, Manizales, Caldas, Colombia. Teléfono: 3175762598

Correo electrónico: jjsepulvedal@unal.edu.co
Historia del artículo:

Artículo recibido: 30-VI-2017/ Aprobado: 26-IV-2017

Disponible online: 3 de agosto 2018

Discusión abierta hasta abril de 2020 
It has been noted that, these two phenomena integrate social and technological factors, that must be considered to implement the programs or projects concerning this matter.

Additionally, the Information Systems Management is a field of study, that comprises the sufficient knowledge to study this type of phenomena, since the management is a social sciences discipline, and the information systems are products developed in the exact sciences field.

In this paper, it is appointed the relation between the Information Systems Management and the study (analysis, improvement, design and proposal) concerning the digital gap and the inclusion gap phenomena.

Keywords: digital inclusión, digital divide, digital gap, information systems

\section{Divisão digital e inclusão digital: fenômenos sócio-tecnológicos}

\section{Resumo}

Existe uma diferença entre as comunidades em relação ao acesso e uso de ferramentas tecnológicas para atender às necessidades e melhorar as condições de vida; fenômeno definido como divisão digital. Para reduzir a diferença, propostas e processos foram feitos dentro do marco do fenômeno com inclusão digital.

Foi identificado que esses dois fenômenos integram aspectos sociais e tecnológicos que devem ser abordados na implementação de programas e projetos.

Além disso, a Administração de Sistemas de Computação é uma área que integra o conhecimento necessário para estudar esses fenômenos. A administração é uma disciplina das ciências sociais e dos sistemas de computação que estão dentro da estrutura das ciências exatas.

Este documento apresenta a relação entre a Administração de Sistemas de Computação e o estudo (análise, melhoria, design e proposta) dos fenômenos de exclusão e inclusão digital.

Palavras-chave: inclusão digital, exclusão digital, sistemas computacionais.

\section{Introducción}

Los productos tecnológicos de información y comunicaciones (TIC) son vistos como herramientas con las cuales, a partir de su acceso y/o uso, se espera mejorar la calidad de vida de las personas y de las sociedades que conforman. A partir de esta premisa se emprenden programas y proyectos cuyo propósito es acercar estos productos a individuos y comunidades ${ }^{1}$ menos favorecidas (según sus con-

1 Entendiendo en este caso como comunidades a cualquier grupo de personas que se configuren en el "objeto" de los procesos para inclusión digital. diciones económicas, educativas y de género, por ejemplo), estas iniciativas se conocen como inclusión digital.

El propósito de la inclusión digital es cerrar lo que se ha denominado la brecha digital, descrita como la diferencia que existe entre aquellas personas que tienen acceso a las TIC y aquellas que no lo tienen. La brecha digital, al igual que el fenómeno de inclusión digital es un sistema complejo y multidimensional, y además sufre procesos de evolución y adaptación en su conceptualización (Sepúlveda López \& Ramírez Castañeda, 2015). 
A pesar de reconocer este aspecto cambiante de los fenómenos, los estudios sobre inclusión digital se han realizado de forma reducida, sin considerar aspectos más allá de la brecha de acceso; que consiste en acercar las herramientas tecnológicas a las personas que aún no las tienen. Para abordar la inclusión y la brecha digital se deben considerar aspectos sociales y tecnológicos de manera simultánea. Estas situaciones no solo se deben analizar desde el acceso a herramientas TIC (aspecto tecnológico), además, se hace necesario considerar características personales y grupales de las comunidades que son "objeto" de los programas y proyectos (aspecto social) que tienen como objetivo la ausencia de acceso, alfabetización y uso efectivo de estos productos. Se considera que la Administración de Sistemas Informáticos (ASI) es un área disciplinar que integra conocimientos necesarios para estudiar la brecha y la inclusión digital. La administración es una disciplina de las ciencias sociales y los sistemas informáticos (metodologías y procesos TIC) son productos que se desarrollan en el marco de las ciencias exactas.

En el presente documento se plantea la relación entre la ASI y el estudio (análisis, mejora, diseño y propuesta) de los fenómenos de la brecha e inclusión digital. El documento está estructurado de la siguiente manera: primero, se revisa el espacio problema de la Administración de Sistemas Informáticos; segundo, se enmarcan la brecha y la inclusión digital en este espacio problema; tercero, se muestra la integración de aspectos sociales y tecnológicos al interior de estos dos fenómenos; y cuarto, se plantean las conclusiones.

\section{Administración de Sistemas Informáticos: integración de conocimientos sociales y tecnológicos}

Para el propósito de este documento, el espacio problema de una disciplina es el sistema n-dimensional en el cual se estudian y analizan situacio- nes, se plantean soluciones y se gestionan proyectos relacionados con un área del conocimiento. El espacio problema de la ASI se empieza a configurar en la década de los setenta del siglo pasado. En este caso particular este espacio se conforma, por el lado de la administración con conocimientos de diferentes ciencias sociales, y del lado de los Sistemas Informáticos con el desarrollo de las ciencias exactas (matemáticas e informática).

La relación entre el desarrollo de las actividades realizadas en las organizaciones y la utilización de productos TIC se da luego del avance aislado de disciplinas relacionadas con los temas administrativos y con los temas tecnológicos. Es así como desde la economía, la psicología, la ingeniería electrónica, la ingeniería de software y las ciencias de la computación, por ejemplo, se toman aportes para la definición y la descripción de la ASI.

En el documento 'Computing Curricula 2005. The Overview Report, Covering undergraduate degree programs in Computer Engineering, Computer Science, Information Systems, Information Technology, Software Engineering' (The Association for Computing Machinery (ACM); The Association for Information Systems (AIS); The Computer Society (IEEE-CS), 2005) se propone una configuración del espacio problema de las disciplinas relacionadas con la computación o la informática. El esquema propuesto tiene dos ejes: en el eje horizontal se indica si la disciplina se desarrolla más en el campo teórico o en el práctico; y en el eje vertical se relacionan temas de interés de la computación (hardware y arquitectura de computadores, infraestructura de sistemas, métodos y tecnologías de software, tecnologías de aplicación y aspectos organizacionales y sistemas de información).

En la Figura 1 se muestra cuál es el ámbito, dentro del espacio problema general en el que se encuentra la ASI. 
Figura 1. Sistemas de información en el espacio problema de la computación

Fuente: (The Association for Computing Machinery (ACM); The Association for Information Systems (AIS); The Computer Society (IEEE-CS), 2005)

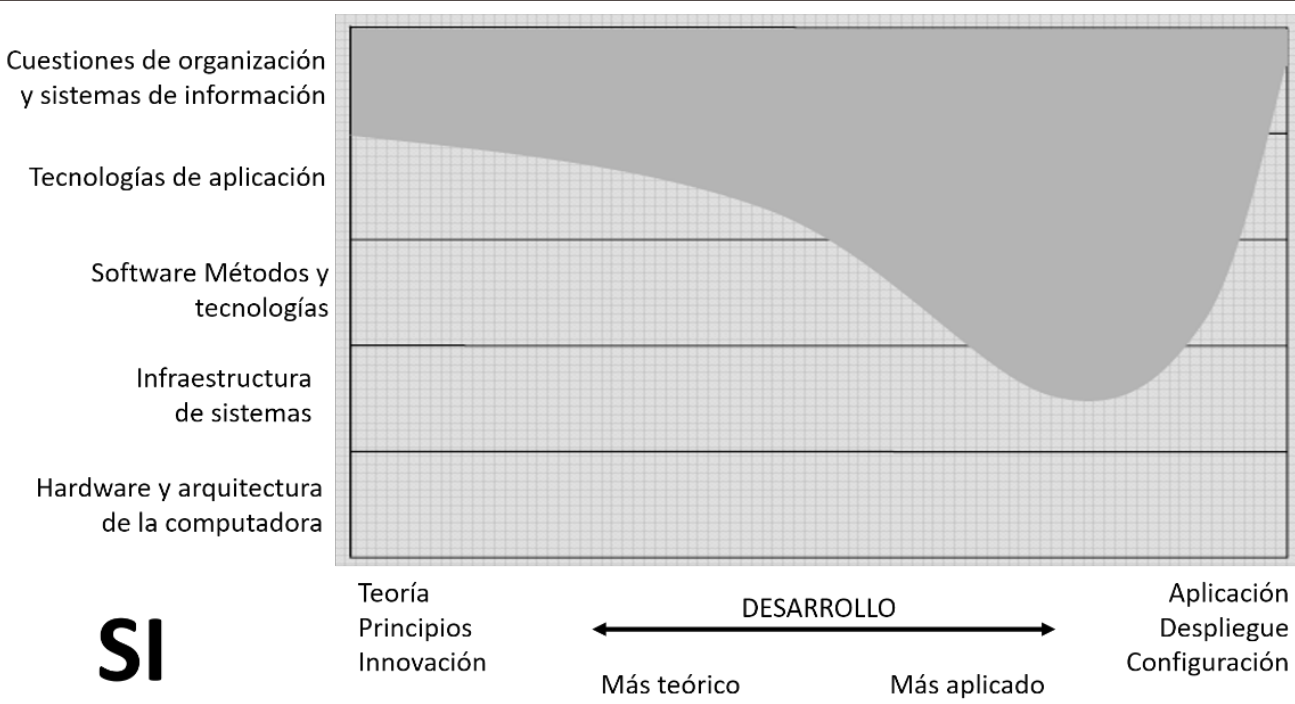

Según el Informe, la ASI se ocupa de propuestas teóricas y prácticas y no se interesa por el hardware y la arquitectura de computadores; en el terreno de lo práctico, estudia en menor medida la infraestructura de sistemas y en mayor los métodos y tecnologías de software y las tecnologías de aplicación; y su ámbito de desarrollo pleno se da sobre aspectos organizacionales y sistemas de información.

La ASI se asocia inicialmente con las actividades de gestión de proyectos de Tecnologías de Información (TI) en empresas tradicionales, sin embargo, es posible ampliar su espacio problema para que considere otro tipo de sistemas empresariales (organizaciones sin ánimo de lucro, informales, públicas y sociales, por ejemplo) y otros productos y servicios tecnológicos (redes de computadores, redes sociales, infraestructuras, etc.). En el documento 'IS 2010. Curriculum Guidelines for Undergraduate Degree Programs in Information Systems' (Association for Computing Machinery (ACM); Association for Information Systems (AIS), 2010) se mencionan otros campos en los que puede 'existir' la ASI: el derecho, la biología y la asistencia médica.
Para ampliar el espacio problema de la ASI se puede considerar tipos diferentes de sociedades: las ciudades, las comunidades físicas y virtuales. De la misma manera, los sistemas informáticos son herramientas que puedan utilizarse para apoyar la estrategia del negocio, administrar las actividades operativas de la organización, posibilitar la proyección de la empresa, respaldar la planeación a corto y largo plazo de la organización, construir comunidades formales e informales, simular su funcionamiento e incluso determinar el impacto del uso de las TIC.

Igualmente, se debe tener en cuenta que los sistemas informáticos son sistemas complejos, ya que relacionan en distintas direcciones y sentidos elementos organizacionales y tecnológicos, en las diferentes organizaciones y sociedades que los utilizan. Además, son analizados, descritos, implementados, utilizados y evaluados por diferentes personas con diversos puntos de vista y perspectivas de la organización y del sistema informático. Cada uno de los integrantes de la organización en la cual se incorpora el uso de TIC tiene una posición específica en la misma, y en esa medida un acceso diferente al 
producto tecnológico, por lo tanto, la funcionalidad que demanda y obtiene del mismo, es distinta.

\section{La inclusión y la brecha digital en el espacio problema de la Administración}

\section{de Sistemas Informáticos}

La ASI es una rama que combina las ciencias sociales (administración) y las ciencias exactas (sistemas informáticos). En este apartado del documento, se muestra la inclusión y brecha digital como áreas de estudio y aplicación de esta disciplina. Para explicar la relación existente, se establece la relación entre la ASI y estos dos fenómenos desde la integración que hacen de aspectos sociales y tecnológicos y desde una revisión conceptual.

Es importante hablar inicialmente de la brecha digital, ya que ésta se establece, antes que la inclusión digital, en el campo práctico y teórico: la problemática de la brecha digital da origen a una serie de procesos para reducirla en el marco de la posterior inclusión digital.

La brecha digital es descrita como la diferencia que existe entre aquellas personas que tienen acceso a las herramientas TIC y aquellas que no. La primera mención del término fue realizada en el año 1995 por la Agencia Nacional de Telecomunicaciones e Información (NTIA), quien la definió como:

"la desigualdad entre los que tienen un ordenador y los que no lo tienen. El interés por el fenómeno estaba relacionado, en ese momento, con las repercusiones que podía suponer para la sociedad norteamericana en el corto y en el medio plazo el hecho constatable de que algunos de sus ciudadanos tuviesen ordenadores y pudiesen, por tanto, acceder a Internet y a sus servicios y contenidos, mientras que otros no podían disponer de ellos, ni acceder a sus beneficios" (Aguntín Lacruz \& Clavero Galofré, 2009).

Se concluye de esta definición, que los estudios que se han hecho del fenómeno se han centrado de forma reducida, al igual que la formulación y aplicación de políticas públicas en el tema, en la brecha de acceso (primera brecha); llevar TIC a las personas menos favorecidas. En otras palabras, "la investigación existente sobre la brecha digital se ha centrado principalmente en la primera brecha" (Brandtzaeg, Heim, \& Karahasanovic, 2011)by using cluster analysis on survey data $(\mathrm{N}=\backslash \mathrm{n} 12,666 /$ age: $16-74$ years. A partir de la definición de la NTIA se presenta una tendencia generalizada de realizar aproximaciones netamente cuantitativas y con énfasis en sus características demográficas a este fenómeno; esta es una aproximación simple al mismo.

Sin embargo, la definición de la brecha digital ha sufrido cambios; uno de los más representativos lo planteó en 2001 la Organización para la Cooperación y el Desarrollo Económico (OCDE), para ésta la brecha digital es el:

"desfase o división entre individuos, hogares, áreas económicas y geográficas con diferentes niveles socioeconómicos con relación tanto a sus oportunidades de acceso a las tecnologías de la información y la comunicación, como al uso de Internet para una amplia variedad de actividades" (Aguntín Lacruz \& Clavero Galofré, 2009).

Por otro lado, la primera mención del termino inclusión digital la hizo la Communication Rights in the Information Society (CRIS) en el año 2003 durante la Cumbre Mundial de la Sociedad de la Información celebrada en Ginebra. Allí la inclusión digital fue definida como el:

"conjunto de políticas públicas relacionadas con la construcción, administración, expansión, ofrecimiento de contenidos y desarrollo de capacidades locales en las redes digitales públicas, alámbricas e inalámbricas, en cada país y en la región entera. Incluye las garantías de privacidad y seguridad ejercidas de manera equitativa para todos los ciudadanos" (Aguntín Lacruz \& Clavero Galofré, 2009). 
En esta primera definición, el origen de la inclusión digital se puede atribuir a la brecha digital, ya que en esta Cumbre se pretendía establecer una solución a esta situación de desigualdad en el acceso a las TIC y proponer los mecanismos para brindarlas de forma equitativa para todos los ciudadanos.

En el fenómeno de la inclusión digital se han identificado una serie de definiciones que contemplan variados aspectos tecnológicos y sociales. Se identifican en las múltiples definiciones relacionadas a continuación, como el concepto de inclusión digital ha trascendido de un carácter cuantitativo simple (aspecto tecnológico) basado en la oferta de acceso a internet y herramientas TIC (definiciones a, d, i) a ser considerado un fenómeno que debe involucrar aspectos de carácter social (definiciones $b$, c, e, f, g, h, j, k).

a. Procesos desarrollados por entidades gubernamentales y no gubernamentales para cerrar la brecha digital y promover la inclusión social y la alfabetización digital (Thompson, et al., 2014).

b. Habilidades de procesamiento de información, comportamiento subcultural y actitud personal son los tres factores que afectan la riquezas o pobreza de información, lo que se refleja directamente en los niveles de inclusión social (Thompson, et al., 2014).

c. Powell, Byrne y Dailey (2010): desde la perspectiva de las personas marginadas, hace relación a la dificultad para obtener y mantener el acceso a la banda ancha, y esfuerzos para tener acceso al empleo, la educación y los servicios públicos (como se cita en Thompson, Jaeger, Greene, Subramaniam, \& Bertot, 2014).

d. Seale, Draffan y Wald (2010): un fenómeno por el cual las personas marginadas son capaces de acceder y participar de manera significativa en las mismas actividades de aprendizaje, de empleo, sociales y de ciudadanía como los demás, esto a través del acceso a las tecnologías digitales (como se cita en Thompson, Jaeger, Greene, Subramaniam, \& Bertot, 2014). e. El IMLS - la agencia de Gobernación federal en los Estados Unidos (2011): la capacidad de los individuos y grupos para acceder y utilizar las tecnologías de información y comunicación. Abarcan no sólo el acceso a Internet, sino también la disponibilidad de hardware y software; contenidos y servicios pertinentes; y capacitación para la alfabetización necesaria para el uso efectivo de las tecnologías de información y comunicación (como se cita en Thompson, Jaeger, Greene, Subramaniam, \& Bertot, 2014).

f. Forma de inserción social imprescindible para el crecimiento comunitario, que surge como superación de la dimensión netamente cuantitativa y tecnológica del concepto de brecha digital (Aguntín Lacruz \& Clavero Galofré, 2009).

g. La medida en que los sectores desfavorecidos de una sociedad pueden tener la misma oportunidad de participar en la sociedad de la información (Thompson, et al., 2014).

h. Martin (2006): la conciencia, la actitud y la capacidad de los individuos para utilizar adecuadamente los servicios digitales para identificar, acceder, administrar, integrar, evaluar, analizar y sintetizar recursos digitales, la construcción de nuevos conocimientos, crear expresiones de medios y comunicarse con los demás, en el contexto de situaciones específicas de la vida, con el fin de permitir una acción social constructiva; y reflexionar sobre este proceso (como se cita en Thompson, Jaeger, Greene, Subramaniam, \& Bertot, 2014).

i. Combinación de servicio suficiente y asequible de banda ancha, la disponibilidad de oportunidades para desarrollar la alfabetización digital necesaria para utilizar la banda ancha (Thompson, et al., 2014).

j. Habilidad de individuos y grupos para acceder y usar las tecnologías de información y comunicación (Caruso, 2014).

k. Cullen et al. (2004): Es el proceso de inclusión social en relación con el objetivo de "una socie- 
dad de la información europea para todos". La definición de la inclusión digital [...] abarca las tres dimensiones de la inclusión social, en la medida en que se refiere a desventajas digitales, oportunidades digitales y empoderamiento digital. Como se cita en (Martínez Cantos, 2013).

Con base en esta revisión conceptual, en la siguiente sección del documento se realiza la revisión en detalle de los fenómenos de la inclusión digital como fenómenos socio-tecnológicos.

\section{Brecha digital e inclusión digital:}

\section{Detalle sobre la integración de aspectos sociales y tecnológicos}

Una conclusión que se puede adelantar a partir de la revisión de la evolución de los diferentes conceptos de la brecha e inclusión digital, es que estos fenómenos consideran características tecnológicas y sociales actualmente. De acuerdo con la consideración previa, su análisis y abordaje requiere de personas con conocimientos y capacidad de trabajo interdisciplinario.

En este apartado del documento, se mostrará cómo estos fenómenos han evolucionado de lo tecnológico a lo social; el enfoque ha cambiado, y con él, el impacto que tienen sobre las comunidades.
Una primera referencia a este aspecto se puede observar en la Figura 2, en la cual, con base en la revisión conceptual se muestra las características iniciales y actuales de la inclusión y brecha digital.

Nuevamente se indica que, los dos fenómenos en su inicio se centraban particularmente en el aspecto tecnológico: instalación de redes de comunicación y entrega de herramientas TIC como solución, por ejemplo. Actualmente, y como se muestra en la Figura 2, estos fenómenos se enfocan en aspectos que van más allá de la tecnología; se incluyen otros elementos que complementan la definición y caracterizan la implementación de proyectos relacionados con el objetivo de mejorar la calidad de vida de las personas en una comunidad determinada.

Con el propósito de seguir ilustrando el cambio conceptual y práctico de la inclusión digital se plantea un concepto propio, elaborado con base en la revisión de otros:

El fenómeno de la inclusión digital es descrito como el grupo de procesos por medio de los cuales se pretende brindar acceso físico, intelectual y de información social a un grupo de personas que posee un grupo de características que deben ser tenidas en cuenta para el buen desarrollo de los programas/proyectos.

Figura 2. Evolución Brecha e Inclusión Digital

Fuente: elaboración propia

\section{Brecha Digital}

Acceso Físico
Características Demográficas $\underset{\begin{array}{l}\text { Garantías de privacidad } \\ \text { Construcción y expansión de contenidos } \\ \text { Redes públicas }\end{array}}{\begin{array}{l}\text { Habilidades } \\ \text { Competencias } \\ \text { Educación } \\ \text { Participación Social }\end{array}} \begin{aligned} & \text { Alfabetización digital } \\ & \text { Inclusión Social } \\ & \text { Crecimiento comunitario } \\ & \text { Acceso físico, intelectual e información social }\end{aligned}$

\section{Inclusión Digital}


Lo que se espera con estos procesos es permitir que las personas obtengan o aumenten su participación en actividades de ciudadanía, sociales, de empleo y de aprendizaje, para de esta forma fomentar el crecimiento comunitario y traducirlo en un promotor de la inclusión social.

Se puede llegar a relacionar la descripción de la actividad de la ASI con la definición de la inclusión digital si se tiene en cuenta que comparten la complejidad como método y característica respectivamente. Asimismo, "la brecha digital no existe en un vacío" (Tien \& Fu, 2008)this study explores four research questions: $(1$, sino en contextos que es necesario e importante comprender para mejorar las aproximaciones que de ella se hagan. Para ampliar la idea presentada se menciona que:

"el éxito de los responsables de la inclusión digital dependerá de su capacidad de hacer encaje de bolillos con las muchas tareas que afrontan, incluidas las operaciones técnicas, la cooperación con las comunidades de usuarios, la creación de servicios, el buen gobierno, y el desarrollo de herramientas colaborativas con la participación del usuario" (López López, 2009).

Para continuar con esta exposición la de relación entre los fenómenos de brecha e inclusión digital con la ASI se menciona que el Admistrador de Sistemas Informáticos:

"es un profesional con conocimientos científicos, humanísticos, técnicos, lógicos y sistémicos que lo capacitan para formular, diseñar, implementar y auditar políticas, estrategias, planes y programas en el campo de los sistemas de información utilizados en las distintas organizaciones. Por consiguiente, debe ser un conocedor crítico de las innovaciones, cambios, tendencias y desafíos tecnológicos relacionados con el desarrollo informático, a fin de que pueda garantizar el eficiente funcionamiento $y$ administración de los recursos informáticos que posee la empresa, además de estar en capacidad de crear y dirigir empresas que aporten soluciones a las necesidades informáticas del entorno, contribuyendo al desarrollo de la sociedad en general" (Universidad Nacional de Colombia, 2017).

Esta cita permite considerar de manera adicional, las habilidades para entender e identificar necesidades y problemas especificos de las comunidades en las cuales se utilizan herramientas TIC. La combinación de estos conocimientos brinda al experto en ASI las competencias para comprender, si en casos especificos la tecnología puede llegar a ser una solución; y de ser así, identificar cuáles y de qué forma lasherramientas TIC son las más indicadas para satisfacer estas necesidades o solucionar estos problemas.

\section{Conclusiones}

El espacio problema de la ASI se conforma, por el lado de la administración con conocimientos de diferentes ciencias sociales, y del lado de los Sistemas Informáticos con el desarrollo de las ciencias exactas (matemáticas e informática); la ASI se configura como una rama interdisciplinar que busca gestionar las herramientas TIC para el cumplimiento de objetivos especificos en organizaciones (en esta caso comunidades).

De igual manera, la brecha y la inclusión digital consideran características tecnológicas y sociales, su análisis y abordaje requiere de personas con conocimientos y capacidad de trabajo interdisciplinario. Sin embargo, los estudios que se han hecho de los fenómenos se han centrado de forma reducida, al igual que la formulación y aplicación de políticas públicas, en la brecha de acceso. En la mayoria de ocasiones, los procesos se realizan sin considerar las características de dichas personas que hacen parte de estas comunidades, sin tener en cuenta que cada miembro posee un acceso diferente al produc- 
to tecnológico, y por lo tanto, la funcionalidad que demanda y obtiene es distinta.

Los administradores de sistemas informáticos han desarrollado habilidades para entender $\mathrm{e}$ identificar necesidades y problemas especificos de las organizaciones en las cuales se requiere la utilización de herramientas TIC: comprender en cuáles casos las herramientas tecnológicas pueden ser el medio para satisfacer dichas necesidades o resolver problemas, la manera de hacerlo y la consideración de las implicaciones sociales que estas decisiones conllevan; todos aspectos necesarios para abordar la brecha y la inclusión digital.

\section{Referencias}

Aguntín Lacruz, M. d. C. \& Clavero Galofré, M., 2009. Indicadores Sociales de Inclusión Digital: Brecha y Particiáción Ciudadana. Derecho, gobernanza y tecnologías de la información en la sociedad del conocimiento, pp. 143-165.

Association for Computing Machinery (ACM); Association for Information Systems (AIS), 2010. 'IS 2010. Curriculum Guidelines for Undergraduate Degree Programs in Information Systems'. s.l.:s.n.

Brandtzaeg, P. B., Heim, J., \& Karahasanovic, A. (2011). Understanding the new digital divide-A typology of Internet users in Europe. INTERNATIONAL JOURNAL OF HUMAN-COMPUTER STUDIES, 69(3), 123-138. http://doi.org/10.1016/j.ijhcs.2010.11.004.

Caruso, S., 2014. Creating Digital Communities: A resource to Digital Inclusion. New York: Nova Science Publishers.

Gonzáles Zabala, M. P. \& Sánchez Torres, J. M., 2013. Análisis de las estrategias del Gobierno colombiano para la inclusión de los ciudadanos en la Sociedad de la Información propuestas desde 2000 hasta 2011. Revista de Estudios Sociales, pp. 133-146.

López López, P., 2009. Inclusión Digital: Un Nuevo Derecho Humano. Educación y Biblioteca, pp. 114-118.

Martínez Cantos, J. L., 2013. La persistencia de la brecha digital de género: Análisis cuantitativo de España y Europa. Madrid: Universidad Complutense de Madrid.

Sepúlveda López, J. J., \& Ramírez Castañeda, L. A. (2015). Digital inclusion and social perspective - Critical discussion based on a systematic review of the literature. In R. L. Isaias P. (Ed.), Proceedings of the 14th International Conference WWW/Internet 2015 (pp. 209-212). IADIS. Retrieved from https:// www.scopus.com/inward/record.uri?eid=2-s2.084958529386\&partnerID=40\&md5=be33c2e9ef7 $774 \mathrm{c} 81018 \mathrm{e} 07821 \mathrm{e} 364 \mathrm{~d} 8$.

The Association for Computing Machinery (ACM); The Association for Information Systems (AIS); The Computer Society (IEEE-CS), 2005. Computing Curricula 2005. The Overview Report, Covering undergraduate degree programs in Computer Engineering, Computer Science, Information Systems, Information Technology, Software Engineering'. Estados Unidos de América: IEEE Computer Society.

Thompson, K. y otros, 2014. Digital Literacy and Digital Inclusion - Information Policy and the Public Library. s.l.:Rowman \& Littkefield.

Tien, F. F. ., \& Fu, T.-T. . c. (2008). The correlates of the digital divide and their impact on college student learning. Computers and Education, 50(1), 421-436. http://doi.org/10.1016/j.compedu.2006.07.005.

Universidad Nacional de Colombia, 2017. ADMINISTRACIÓN DE SISTEMAS INFORMÁTICOS. [En línea] Available at: http://www.manizales.unal.edu.co/ menu/programas-academicos/carreras/administracion-de-sistemas-informaticos/

\section{PARA CITAR ESTE ARTÍCULO TO REFERENCE THIS ARTICLE / PARA CITAR ESTE ARTIGO /}

Ramírez Castañeda, L.A.; Sepúlveda López, J.J. (2018). Brecha digital e inclusión digital: fenómenos socio - tecnológicos. Revista EIA, 15(30), julio-diciembre, pp. 89-97. [Online]. Disponible en: https://doi.org/10.24050/reia.v15i30.1152 